\title{
Influence of real photon diffraction on parametric X-ray radiation angular distribution in thin perfect crystals
}

\author{
Yu.A. Goponov, S.A. Laktionova, O.O. Pligina, M.A. Sidnin, I.E. Vnukov* \\ Belgorod National Research University, Belgorod, Russia
}

\section{A R T I C L E I N F O}

\section{Article history:}

Received 1 December 2014

Received in revised form 21 March 2015

Accepted 24 March 2015

Available online 9 April 2015

\section{Keywords:}

Parametric X-ray radiation

Diffracted transition radiation

Diffracted bremsstrahlung

Measurements of an electron beam

parameters

\begin{abstract}
A B S T R A C T
Using the previously proposed method of calculating diffracted photon yields in thin perfect crystals, analyzed a relative contribution of parametric X-ray radiation and diffracted photons in thin crystals. It is shown that for average energy of electrons and the center of the PXR spot diffracted real photon contribution is comparable to the yield of parametric X-ray radiation and determines the shape of the angular distribution of the total emission in this range of observation angles. The possibility of estimating electron beams parameters based on the results of PXR angular distribution measurements is discussed. It is shown that for energy of electrons by far larger than $1 \mathrm{GeV}$ yield of diffracted transition radiation in narrow angular cone becomes predominating and determines the shape of the angular distribution of total emission in the center of radiation spot.
\end{abstract}

(c) 2015 Published by Elsevier B.V.

\section{Introduction}

Parametric X-ray radiation (PXR) generated by passing fast charged particles through crystals had been actively studied till the end of the last century (see, for example, [1,2] and references therein). The interest to this type of radiation was mainly due to search for tunable compact sources of intensive X-ray radiation for practical, especially, medical applications as an alternative to storage rings. Nowadays it is considered to be established (see, for example, [3]), that radiation intensity, obtained through this mechanism of generation and perfect crystals, is not sufficient for actual medical applications.

In the first approximation PXR can be considered as coherent scattering its own electromagnetic field of a particle on the electron shells of periodically arranged atoms of a target [4,5]. By analogy with X-ray diffraction in crystals, there are two approaches to a description of PXR. The so-called kinematic approach suggests that the multiple reflection of PXR photons at the crystal planes is negligible. If the probability of the process is not small, the dynamic theory must be used. As it was noted in [6] and confirmed in a number of experimental works (see, for example, [7] and references therein) kinematic theory of PXR describes the results of emission yield measurements for the energies of electrons from a few $\mathrm{MeV}$ to several $\mathrm{GeV}$ with an accuracy better than $10-15 \%$.

\footnotetext{
* Corresponding author.

E-mail address: vnukov@bsu.edu.ru (I.E. Vnukov).
}

In connection with the agreement between PXR measurements and calculation results recently there were suggested to use PXR in thin crystals for diagnostic of electron beams parameters, in particular, their transverse sizes [8,9]. The results of PXR measurements for thin crystals $[9,10]$ was not well described by the PXR kinematic theory [1,2]. For fast electrons PXR is always accompanied by radiation diffracted in the crystal which is born directly inside the target or on its surface [11,12]. In the first case we can talk about diffracted bremsstrahlung (DB) and in the second one about diffracted transition radiation (DTR). The first is dominated by under the condition $\omega \gg \gamma \omega_{p}$, where $\omega$ is photon's energy, $\gamma$ - Lorentz factor, and $\omega_{p}$ - plasma frequency of the medium, and the second under opposite condition. If condition $\omega \sim \gamma \omega_{p}$ is true, the contributions of both mechanisms of radiation are observed.

The last condition is satisfied in the above mentioned papers devoted to analysis of possibility determination of electron beam parameters on the measured PXR angular distributions [8,9]. Another feature of these works is the use of devices that measure the total characteristics of radiation; for example, the ProxiVision HR25 X-ray camera [13] in the experiment [9] and the positionsensitive detector based on a so-called imaging plate (IP) [14] in the experiment [10]. Therefore, as it was noted in [15] for analysis of these studies results and comparison with results of calculations it is necessary to summing over all orders reflection intensity taking into account characteristics of the used experimental equipment. Availability of experimental data devoted to the electron beam size measurements by means of PXR angular distribution one which are not described theoretically excluding diffraction of 
real photons, provide an opportunity to consider the study of the influence of real photons diffraction on the observable emission characteristics in wide range of electron energy up-to-date and of importance.

\section{Theoretical considerations}

In the experiment, all mechanisms generation of radiation at the Bragg angles are implemented simultaneously, so here we introduce the basic formulae and approaches for each of them that we used for calculation, following mainly the works $[12,15]$. As it was noted in the introduction, kinematic PXR theory describes quite well results of measurements so to calculate the PXR yield we used formula of PXR spectral-angular distribution obtained in the kinematic approximation in the work [16]:

$$
\begin{aligned}
& \frac{d^{2} N}{d Z d \Omega}=\frac{\sum_{\alpha} \alpha \omega^{3}\left|\chi_{\vec{g}}\right|^{2}}{2 \pi \varepsilon_{0}^{3 / 2} \beta\left(1-\sqrt{\varepsilon_{0}} \vec{\beta} \vec{n}\right)} . \\
& {\left[\frac{(\omega \vec{\beta}-\vec{g}) \vec{e}_{\vec{k} \alpha}}{\left(\vec{k}_{\perp}+\vec{g}_{\perp}\right)^{2}+\frac{\omega^{2}}{\beta^{2}}\left\{\gamma^{-2}+\beta^{2}\left(1-\epsilon_{0}\right)\right\}}\right]^{2} .}
\end{aligned}
$$

The system of units used is $\hbar=m_{e}=c=1$. Here, $\epsilon_{0}=1-\omega^{2} / \omega_{p}^{2}, \vec{\beta}=\beta \vec{n}_{0}$ is the electron velocity vector, $\vec{n}_{0}$ and $\vec{n}$ are the individual vectors corresponding to the incoming electron and emitted photon (with the energy $\omega$ and momentum $\vec{k}$ ), $\vec{g}$ is the reciprocal lattice vector, $\vec{e}_{\overrightarrow{k \alpha}}$ are the polarization vectors, $\perp$ is the index denoting the vector projection on the plane normal to $\vec{n}_{0}$. The other notation is conventional. The term $\left|\chi_{\vec{g}}\right|^{2}$ denotes the following value:

$\left|\chi_{\vec{g}}\right|^{2}=|S(\vec{g})|^{2} \exp (-2 W)\left[-\frac{\omega_{p}^{2}}{\omega^{2}} \frac{f(\vec{g})}{Z}\right]^{2}$.

In the expression (2) $|S(\vec{g})|^{2}$ is the structure factor, $\exp (-2 \mathrm{~W})$ is the Debye-Waller factor, $F(\vec{g})$ is the Fourier component of spatial distribution of electrons in a crystal atom $(F(0)=Z$, where $Z$ is number of electrons in an atom).

The account for dependence of the spectral-angular distribution of PXR from angle between direction motion of electron and plane of crystal is based on approach proposed in [17] which is introduced to simplify calculation multiple coordinate systems connected with direction of the primary electron beam (a laboratory system), direction of the crystal plane (a crystal system) and direction of motion of the emitted photon (a coordinate system of the detector).

Transition from coordinates of crystal system and detector one where the respective values are described in a most simply way into the laboratory coordinate system, where they are used for calculation, are performed by corresponding transformation matrix. The divergence of the electron beam incident on the target, multiple scattering of the electrons in the crystal, collimation of radiation and other experimental factors were taken into account according to the procedure described in [18].

For medium-energy electrons radiation in X-ray range of photon energy $(\omega \leqslant 100 \mathrm{keV})$ except PXR, is generated through the mechanisms of bremsstrahlung and transition radiation (TR). Spectral angular distribution of the TR intensity on vacuum environment without taking into account polarization of the radiation can be represented as [19]:

$\frac{d^{2} I_{T R}}{d \omega d \theta}=\frac{2 e^{2} \theta^{3}}{\pi}\left|\frac{1}{\theta^{2}+\gamma^{-2}}-\frac{1}{\theta^{2}+\gamma^{-2}+\omega_{p}^{2} / \omega^{2}}\right|^{2}$, where $\theta$ is a photon emission angle according to the direction of the electron motion.

For simplicity it can be assumed that the TR is generated directly at the inlet into crystal and then it is diffracted therein.

It is known (see, for instance $[20,21]$ ), that the bremsstrahlung in a dense medium in frequency range $\omega \leqslant \gamma \omega_{p}$ is suppressed due to the effect of medium polarization, and degree of the suppression depends on both energies of photon and electron, and angle of a photon emission. While calculating, we used expression for spectralangular distribution of soft component bremsstrahlung $\left(\omega \ll E_{e}\right)$, obtained in [22], which takes the suppression into account.

$\frac{d^{2} I_{B S}}{d \omega d \Omega}=\frac{\gamma^{2}}{\pi L} \frac{\left(1+\frac{\gamma^{2} \omega_{p}^{2}}{\omega^{2}}\right)^{2}+\gamma^{4} \theta_{\gamma}^{4}}{\left(1+\frac{\gamma^{2} \omega_{p}^{2}}{\omega^{2}}+\gamma^{2} \theta_{\gamma}^{2}\right)^{4}}$

where $\mathrm{L}$ - the radiation length.

Angle of a photon emission in expressions (3) and (4) is measured from the direction of motion of the emitting electron. Whereas in the experiments it is generally measured dependence of the resulting radiation yield $Y(\omega, \theta)$ in the collimator aperture $\vartheta_{c}$ on the observation angle $\theta$ with respect to the center of the reflex, located at an angle $\Theta_{D}$.

To obtain such information it is necessary to take into account scattering of electrons inside the target. To calculate spectral-angular distribution of bremsstrahlung with account multiple scattering in accordance with the methodology [18], the target is divided into a large number of layers with thickness $\Delta t$.

The angular distribution of electrons $P\left(t, \theta_{e}, \varphi_{e}\right)$ after $i$ th layer is calculated by convolution of the multiple scattering angular distribution of particles in this layer with distribution of electrons entering into it. It was assumed that the angular distribution of electrons after passing substance layer of thickness $\Delta t$ is described by Moliere distribution [23].

In this case, the spectral-angular distribution of bremsstrahlung born at a given depth $t$ inside the crystal, $\frac{d^{2} I_{B S}^{*}(\omega, \theta, \varphi, t)}{d \omega \Omega}$ is defined by convolution of the spectral-angular distribution of bremsstrahlung with the angular distribution of electrons at this depth and may be written as:

$$
\begin{gathered}
\frac{d^{2} I_{B S}^{*}(\omega, \theta, \varphi, t)}{d \omega d \Omega}=\int P\left(t, \theta_{e}, \varphi_{e}\right) d \Omega_{e}, \\
\int \frac{d^{2} I_{B S}\left(\omega, \theta_{\gamma}, \varphi_{\gamma}\right)}{d \omega d \Omega_{\gamma}} f\left(\vec{n}, \vec{n}_{e}, \vec{n}_{\gamma}\right) d \Omega_{\gamma} .
\end{gathered}
$$

Here $\vec{n}_{e}\left(\theta_{e}, \varphi_{e}\right)$ and $\vec{n}_{\gamma}\left(\theta_{\gamma}, \varphi_{\gamma}\right)$ are vectors describing the propagation direction of the electron and photons in the laboratory frame of reference and in the system connected with the direction of electron motion, respectively. $\vec{n}(\theta, \varphi)=\vec{n}_{e}+\vec{n}_{\gamma}$ is a vector direction of the photon in the laboratory frame, and $f\left(\vec{n}, \vec{n}_{e}, \vec{n}_{\gamma}\right)$ is a function describing connection between these vectors, see [24] for details.

With this in mind angular distribution of the diffracted radiation relative to the center of reflex along axis $x$ (see, for example, [11]) can be represented as:

$Y_{D B}\left(\omega, \theta_{x}\right)=\int_{0}^{T} d t \int d \omega$

$\int \frac{d^{2} I_{B S}^{*}}{d \omega d \Omega} R\left(\omega, \vec{n}, \vec{g}, \Theta_{D}\right) S(\omega, \vec{n}, t) d \Omega$

$Y_{\text {DTR }}\left(\omega, \theta_{x}\right)=\int d \omega$ 
$\int \frac{d^{2} I_{T R}^{*}}{d \omega d \Omega} R\left(\omega, \vec{n}, \vec{g}, \Theta_{D}\right) S^{*}(\omega, \vec{n}, T) d \Omega$,

where $\frac{d^{2} I_{T R}^{*}}{d \omega d \Omega}$ is the spectral-angular distribution of the transition radiation, taking into account the divergence of primary electron beam, calculated by analogy with the expression (5), $R\left(\omega, \vec{n}, \vec{g}, \Theta_{D}\right)$ is the reflectivity for these directions of the vectors $\vec{n}$ and $\vec{g}$, defined crystal orientation angle $\Theta$ and location of the detector $\Theta_{D}$. $S^{*}(\omega, \vec{n}, T)$ and $S(\omega, \vec{n}, t)$ are functions taking into account photon absorption in the crystal and geometry of the experiment, Tis the crystal thickness. The integration into (6) and (7) is taking place over all angles and photon energies with the hit of photons in the collimator.

If necessary, for consideration of the spatial electron beam distribution on the crystal (see below) in the expression for the observed angular distribution of radiation (6) and (7) may be included additional integration over coordinates of radiation point taking into account this spatial distribution.

To determine the yield of the diffracted radiation it is necessary to know reflectivity of the crystal $R\left(\omega, \vec{n}, \vec{g}, \Theta_{D}\right)$. We used method described in [15]. This method based on the approach proposed in the article [12] and allows to take into account multiple Bragg re-scattering, absorption and scattering photons due to processes that are not associated with diffraction. The used technique [15] is not limited concerning thickness and geometry of a using crystal. Provided some modifications it can be used for more complex functional dependence of reflection probability on the angle between the direction of the photon movement and the plane of the crystal than it was used in [12].

\section{Relative contributions of PXR and real diffracted photons in thin crystals}

As is noted in the introduction, main purpose of this study is explain the results of new experiments, where the PXR kinematic theory does not describe the measurements results without considering the contribution of real photons diffraction and analysis of possibility to use similar measurements for estimation of the electron beam parameters. In order to analyze the influence of the diffracted real photons contribution and experimental conditions on the angular distribution of the resulting radiation a series of radiation yield calculations for the experimental conditions [10] were performed with procedure [15]. Electron beam energy of $255 \mathrm{MeV}$ with divergence $\vartheta_{e} \sim 0.25 \mathrm{mrad}$ hits on a silicon crystal thickness of $20 \mu \mathrm{m}$. Reflection (220) is being investigated. The system of detecting is located at a distance of $1 \mathrm{~m}$ from a crystal at an angle $\Theta_{D}=2 \Theta_{B}=32.2^{\circ}$. The square detector the size of which is $0.3 \times 0.3 \mathrm{~mm}^{2}$ is moving down through the center of reflex with a step of $0.3 \mathrm{~mm}$.

Fig. 1 shows vertical angular distribution of parametric X-ray radiation - points. Distributions of the diffracted transition radiation and the diffracted bremsstrahlung and total radiation yield for experimental conditions [10] and the first reflections order calculated by a technique [15], so these are the curves 1-3, respectively. The results of PRX calculations excluding divergence of electron beam and multiple scattering of electrons within the crystal are shown also (curve 4).

As can be seen from the figure, PXR possesses bigger intensity than the diffracted radiation, and its angular distribution is significantly broader. In the center of the PXR angular distribution there is a dip, whereas output of the diffracted radiation is concentrated near Bragg's direction. As a result, output of the diffracted photons gives the main contribution into output of radiations in the center of the reflex as it was observed in the experiments [11,12]. It should be noted that multiple scattering of electrons in crystal

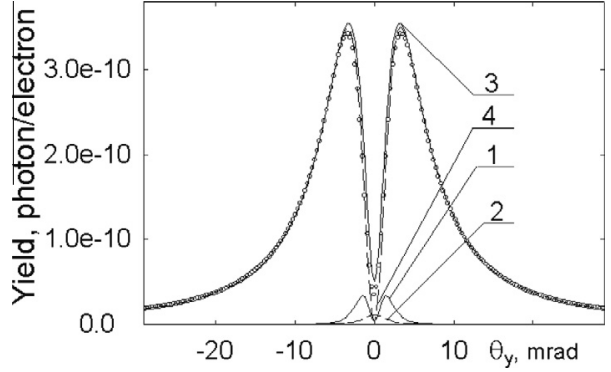

Fig. 1. Vertical distribution of the $X$-ray yield for [10] condition and the first reflection order. $\circ$ - PXR; 1 - DTR; 2 - DB; 3 - PXR + DTR + DB; 4 - PXR without the electrons multiple scattering and the electron beam divergence.

and electron beam divergence which are not taken into account while comparing calculated and experimental results in the [10], see the dependence of 4 , also increase the radiation intensity in the center of PXR reflex.

For the experimental conditions [10] and the first reflection order the condition $\omega \sim \gamma \omega_{p}$ is satisfied, so we can observe both DB and DTR. Energy of photons of the first allowed reflection order $\omega=11.65 \mathrm{keV}<\gamma \omega_{p} \approx 15.97 \mathrm{keV}$, that is why the yield of diffracted bremsstrahlung is suppressed due to the Ter-Mikaelian effect of density [20,21], and appears to be less than the DTR yield.

For using of PRX for diagnostic of electron beams parameters authors of $[8,9]$ assume to register radiation not for individual reflection orders, but the angular distribution of the resulting radiation, since this approach requires significantly less time for information receiving. That is why let us analyze the differences between the resulting angular distribution (o) and the distributions for individual orders of reflection shown in Fig. 2 for experimental conditions [10] and three orders of reflection, these are the curves $1-3$, respectively.

The figure shows that the main contribution to the total distribution gives the first order of reflection. Contribution of the second and especially the third orders with higher energy of photons, and more narrow angular distribution is substantially less. However, the second-order contribution is sufficient enough to be noticed, and while using the absorber on the way of the photon beam from the crystal to the detector, as, for example, in the experiment [9] can be comparable with the contribution of the first reflection order.

As is noted in the introduction, authors of the above cited works $[8,9]$ proposed to use measurements of the total radiation angular distribution for determination of an electron beam size. To test the hypothesis about influence of this factor on the total emission angular distribution it was made calculation taking into account spatial distribution of the electron beam on the crystal according with information listed in [10].

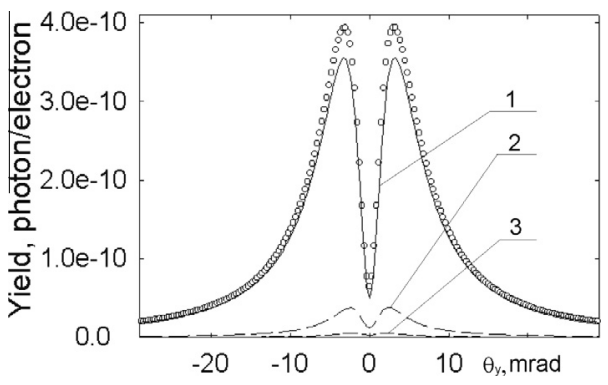

Fig. 2. Vertical distribution of the X-ray yield for [10] condition and three reflection orders. 


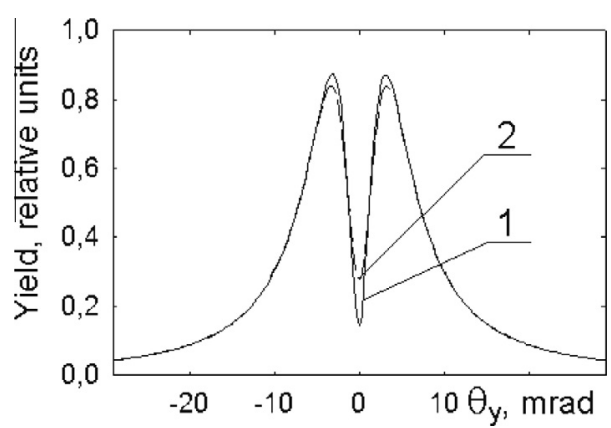

Fig. 3. Vertical angular distribution of total radiation for the experiment [10]. 1 calculation for point like electron beam; 2 - calculation taking into account transverse sizes of the beam.

Fig. 3 shows results of calculation of the vertical angular distribution of radiation from a silicon crystal obtained by means of IP in the experiment [10]. The dependence 1 presents the calculated angular distribution of resulting radiation PXR $+D T R+D B$, obtained taking into account dependence of the IP response from photon energy and quanta absorption on the way from the crystal to the IP, see [25] for details. Sizes of the electron beam on the crystal are not taken into account. The curve 2 in the figure is simulation results taking into account the dimensions of the electron beam on the crystal $\sigma_{x} \approx 0.2 \mathrm{~mm}$ and $\sigma_{y} \approx 0.7 \mathrm{~mm}$ (one standard deviation). During the simulation it was assumed that the coordinate of the emitting electron is distributed over two-dimensional Gauss distribution with the experimentally measured $\sigma_{x}$ and $\sigma_{y}$ [10]. All other calculations were conducted according to the procedure described in [15].

From the figure one can see that when the spatial dimensions of the electron beam on the crystal is taken into account the "bottom" of the distribution hole is lifted and the distribution maxima become slightly lower. For other parts of both distributions the difference between the dependencies is almost negligible. Therefore, under using of large distances between the crystal and the coordinate-sensitive detector for measurement of the transverse size of the electron beam as in the experiment [8] only the central part of the reflex should be analyzed. Direct contribution of the spatial dimensions of the electron beam on the crystal into the PXR angular distribution measured may be observed for very small distance between the crystal and the detector only and comparatively large the electron beam size as in the experiment [9].

The kinematic PXR theory was experimentally confirmed for electrons energy about $1 \mathrm{GeV}$ and less and crystal thickness about some tens microns and larger. Contribution of the diffracted photons into the total radiation yield was observed for the same energy range of electrons $[11,12,25]$. However we can calculate contribution of diffracted real photons into total radiation yield for larger energy of electrons also. It is very interesting because the authors of [9] and other similar works propose to use the PXR angular distribution measurements for estimation of electron beam size of high energy electron accelerators.

Fig. 4 presents results of this calculation for [9] experimental condition and the first reflection order. Electron beam hits on a silicon crystal thickness of $50 \mu \mathrm{m}$. Reflection (220) is being investigated. The system of detecting is located at a distance of $1 \mathrm{~m}$ from the crystal at an angle $\Theta_{D}=2 \Theta_{B}=22.5^{\circ}$. The square detector the size of which is $0.05 \times 0.05 \mathrm{~mm}^{2}$ is moving down through the center of reflex with a step of $0.05 \mathrm{~mm}$. Curves 1 and 2 are calculation results for PXR and DTR angular distributions for electron energy of $1 \mathrm{GeV}$. Curves 3 and 4 are the same dependencies for electron energy of $10 \mathrm{GeV}$. The $\mathrm{DB}$ contribution is negligible and not

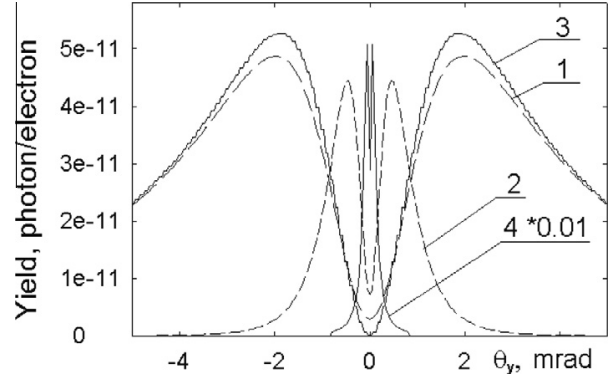

Fig. 4. Vertical distribution of the X-ray yield for [10] condition and the first reflection order. 1 - PXR for $E_{0}=1 \mathrm{GeV} ; 2$ - DTR for $E_{0}=1 \mathrm{GeV} ; 3$ - PXR for $E_{0}=10 \mathrm{GeV} ; 4-$ DTR for $E_{0}=10 \mathrm{GeV}$.

presented because the photon energy $\omega=16.55 \mathrm{keV} \ll \gamma \omega_{p} \approx$ $60 \mathrm{keV}$ and $600 \mathrm{keV}$ for electron energy of $1 \mathrm{GeV}$ and $10 \mathrm{GeV}$, respectively. Relatively large DTR contribution in comparison with results for electron energy of $255 \mathrm{MeV}$ (see Fig. 1) is connected with the increasing of the particles energy (see below) and a smaller size of the detector.

From the figure one can see that increasing of the electron energy practically does not change the PXR angular distribution. The small difference between these distributions is connected with less electron multiple scattering in the crystal for larger energy of electrons. For DTR angular distributions this difference is very large because the transition radiation intensity is proportional to the energy of electron and the TR angular cone characteristic size is about $\gamma^{-2}$. Hence if the kinematic PXR theory and our approach are valid for electron energy of $10-100 \mathrm{GeV}$ the total emission angular distribution will have another form: smooth pedestal and a narrow bright peak with by far larger intensity than the pedestal one.

This result allows to propose another method of electron spatial distribution measurement than in the above cited works. We can measure the central part of the radiation distribution only and used the detector placed at the distance about $1 \mathrm{~m}$ or larger. In this case PXR contribution will be a rather small and may be taken into account by means of calculation. Influence of transition radiation angular distribution for electron energy of $10 \mathrm{GeV}$ or larger will be compared with the spatial electron beam size and may be taken into account by means of calculation also.

\section{Summary and conclusions}

Results of the study may be briefly stated as follows:

(1) The contribution of diffraction of real photons is maximum for thin crystals, where it is manifested in the "swelling" of the minimum in the orientation dependence or angular distribution and may be compared with PXR yield in this point.

(2) The results of measurements of the angular distributions of the total radiation of fast-moving electrons in thin crystals may be used to determine transverse dimensions of electron beams. For large distances between the crystal and the coordinate sensitive detector this information may be obtained from the analysis of the deep in the radiation angular distribution.

(3) For electron energies larger than $10 \mathrm{GeV}$ the results of measurements of the angular distributions of the resulting radiation of fast-moving electrons in thin crystals may be used to determine transverse dimensions of electron beams also. In this case we should analyze the maximum in center of the total emission spot only. For using of this method the 
kinematic PXR theory and the method of DTR angular distribution calculation should be experimentally checked and may be improved for these energies of electrons.

\section{Acknowledgements}

The authors wish to thank the co-authors [12] for their help in the development and implementation of the methodologies used in the research process. The work was supported by the Ministry of Education and Science of the Russian Federation (the State assignment N 3.500.2014/K).

\section{References}

[1] V.G. Baryshevsky, I.Y. Dubovskaya, Itogi Nauki Tekh., Ser. Beams of Charged Particles and Solid Body, vol. 4, VINITI, Moscow, 1991. p. 129 (in Russian).

[2] R. Rullhusen, X. Artru, P. Dhez, Novel Radiation Sources Using Relativistic Electrons, World Scientific, Singapore, 1999.

[3] J. Freudenberger, E. Hell, W. Knupher, Nucl. Instr. Methods A 466 (2001) 99.

[4] G.M. Garibyan, C. Yang, Sov. Phys. JETP 34 (1972) 495.

[5] V.G. Baryshevsky, I.D. Feranchuk, Sov. Phys. JETP 34 (1972) 502.

[6] H. Nitta, Nucl. Instrum. Methods B 115 (1996) 40.
[7] K.-H. Brenzinger, B. Limburg, H. Backe, et al., Phys. Rev. Lett. 79 (1997) 2462.

[8] Y. Takabayashi, Phys. Lett. A 376 (2012) 2408.

[9] G. Cube et al., in: Proceedings of IPAC2013, Shanghai, China, p. 491

[10] Y. Takabayashi, A.V. Shchagin, Nucl. Instrum. Methods B 278 (2012) 78.

11] K.-H. Brenzinger, C. Herberg, B. Limburg, et al., Z. Phys. A 358 (1997) 107.

[12] A.N. Baldin, I.E. Vnukov, B.N.Kalinin, E.A. Karataeva, Poverkhnost' No. 4, 2006 p. 72 (in Russian).

[13] High-Resolution X-Ray Camera, <http://www.proxivision.de/datasheets/ X-Ray-Camera-HR25-X-ray-PR-0055E-03.pdf> (02/092013).

[14] A.L. Meadowcroft, C.D. Bentley, E.N. Stott, Rev. Sci. Instr. 79 (2008) 113102.

[15] S.A. Laktionova, O.O. Pligina, M.A. Sidnin, I.E. Vnukov, J. Phys.: Conf. Ser. 517 (2014) 012020, http://dx.doi.org/10.1088/1742-6596/517/1/012020.

[16] H. Nitta, Phys. Lett. A 158 (1991) 270.

[17] A. Potylitsin, v1, 1998. <arXiv:cond-mat/9802279>

[18] E.A. Bogomazova, B.N. Kalinin, G.A. Naumenko, et al., Nucl. Instrum. Methods B 201 (2003) 276.

[19] V.A. Bazylev, N.K. Zhevago, Radiation of Relativistic Particles in External Fields and in Matter, Nauka Pub, Moscow, 1987 (in Russian).

[20] M.L. Ter-Mikaelian, High-Energy Electromagnetic Processes in Condensed Media, Wiley-Interscience, New York, 1972.

[21] P.L. Anthony et al., Phys. Rev. Lett. 76 (19) (1996) 3350.

[22] V.P. Kleiner, N.N. Nasonov, N.A. Shlyahov, Ukr. Phys. J. 37 (1992) 48 (in Russian).

[23] H.A. Bethe, Phys. Rev. 89 (6) (1953) 1256.

[24] I.E. Vnukov, B.N. Kalinin, A.P. Potylitsin, Sov. Phys. J. 34 (1991) 481.

[25] Yu. A. Goponov et al., Nucl. Instrum. Methods B, submitted for publication. 